\title{
Activities of Serum Ferritin and Treatment Outcomes Among COVID-19 Patients Treated With Vitamin C and Dexamethasone: An Uncontrolled Single-Center Observational Study
}

Hemanth Reddy Burugu ${ }^{1}$, Venkataramana Kandi ${ }^{2}$, Lakshmi Venkata Simhachalam Kutikuppala ${ }^{3}$, Tarun Kumar Suvvari ${ }^{4}$

1. Medicine, Prathima Institute of Medical Sciences, Karimnagar, IND 2. Clinical Microbiology, Prathima Institute of Medical Sciences, Karimnagar, IND 3. Medicine, Konaseema Institute of Medical Sciences and Research Foundation (KIMS \& RF), Amalapuram, IND 4. Public Health, Rangaraya Medical College, Kakinada, IND

Corresponding author: Lakshmi Venkata Simhachalam Kutikuppala, simhachalam.kutikuppala@gmail.com

\section{Abstract}

\section{Background}

The fatal outcomes by COVID-19 are accompanied by cytokine storm syndrome, and thereby it is reported that disease severity is dependent on the cytokine storm syndrome. This cytokine storm can be assessed by evaluating the serum ferritin levels. The objective of this study was to assess the activities of serum ferritin and treatment outcomes among COVID-19 patients who were treated with dexamethasone and vitamin C.

\section{Materials and methods}

A single-center, prospective, observational study was conducted among SARS-CoV-2 infected patients from July 2020 to August 2020. The diagnosis was confirmed by real-time polymerase chain reaction (RT-PCR) and computed tomography (CT) imaging of the lungs. Serum ferritin levels were compared with the treatment outcomes of COVID-19 positive patients who were treated with dexamethasone and vitamin C.

\section{Results}

A total of 50 COVID-19 patients were included in the study. The mean age was 41.70 years. The recovery rate (94\%) was remarkably high and is a good sign of COVID 19 treatment with vitamin C and dexamethasone as key modalities. The mean serum ferritin levels among recovered and expired patients were $478.81 \mathrm{ng} / \mathrm{ml}$ and $1410 \mathrm{ng} / \mathrm{ml}$, respectively.

\section{Conclusion}

The serum activities of ferritin were markedly increased in COVID-19 patients who could not survive as compared to the patients who finally recovered from the infection.

Review began 10/27/2020 Review ended 11/02/2020 Published 11/11/2020

\section{() Copyright 2020}

Burugu et al. This is an open access article distributed under the terms of the Creative Commons Attribution License CC-BY 4.0., which permits unrestricted use, distribution, and reproduction in any medium, provided the original author and source are credited.
Categories: Allergy/Immunology, Infectious Disease, Pulmonology

Keywords: covid-19, dexamethasone, vitamin c, serum ferritin, ct-imaging, lungs, sars-cov-2

\section{Introduction}

COVID-19 caused by SARS-CoV-2 was declared as a public health emergency on January 30, 2020. The outbreak was declared as a pandemic on March 11, 2020, by the World Health Organization (WHO). The primary mode of spread is through close contact and via respiratory droplets produced from coughs or sneezes. The SARS-CoV-2 main target is binding to the angiotensin-converting enzyme 2 (ACE2) receptors, which are most commonly present in the lung, kidney, and gastrointestinal tract [1].

SARS-CoV-2 infection, especially in older patients and those with pre-existing illness, can progress to severe disease with critical respiratory symptoms [2]. Therefore, early recognition of severe forms of COVID-19 is essential for the timely triaging of patients [2]. Ferritin is a crucial mediator of immune dysregulation, contributing to the cytokine storm under extreme hyperferritinemia via direct immune-suppressive and proinflammatory effects [3].

Elevated levels of ferritin, or hyperferritinemia, indicate the viral or bacterial load in the body. Hyperferritinemia, or hyperferritinemic syndrome, is a condition activating macrophages to secrete cytokines, causing a cytokine storm in severe cases, which can be a sign of severe disease [4]. The fatal outcomes by COVID-19 are accompanied by cytokine storm syndrome. It is thereby reported that disease severity depends on the cytokine storm syndrome. This cytokine storm can positively be assessed by evaluating the serum ferritin levels [5]. 
Ideally, serum ferritin levels might be a crucial factor influencing the severity of COVID-19. Many individuals with diabetes exhibit elevated serum ferritin levels, facing a higher probability of experiencing severe complications from COVID-19 [6]. The median values of serum ferritin levels from a few recent studies exceeded the upper limit of detection in the COVID-19 patients during all the days of hospitalization, suggesting that ferritin levels increased incessantly throughout the hospital stay [7].

In this study, we assessed the correlation between serum ferritin and severity and mortality of COVID-19 and treatment outcome with dexamethasone and vitamin C in COVID-19 positive patients. We aimed to evaluate the serum concentrations of ferritin and correlate it with the clinical outcomes.

\section{Materials And Methods}

A single-center, prospective, observational study was conducted among COVID-19 positive patients at a tertiary care hospital from July 2020 to August 2020 (2 months). All the patients included in the study were real-time polymerase chain reaction (RT-PCR) positive and had typical Computed tomography (CT) radiological features suggestive of COVID-19. The age range of the patients included in the study was between 25-50 years and written informed consent was obtained from all the study participants and confidentiality of information was assured to them. This study was approved by the Institutional Ethical Committee of the Prathima Institute of Medical Sciences, Telangana. The patients who had severe comorbidities and were critically ill at the time of presentation and patients below 25 or above 50 years of age were excluded from the study.

The socio-demographic and clinical profile data were collected as shown in Table 1. A blood sample was collected from all the patients and sent for laboratory investigations; exclusively serum ferritin levels were compared with the treatment outcomes of all the patients who were treated with dexamethasone and vitamin C. The serum ferritin level was determined by chemiluminescence immunoassay (CLIA).

\section{Statistical analysis}

The data were expressed as mean or percentage. We compared the categorical variables between the two groups using Pearson's chi-square test. A p-value of $<0.05$ was considered statistically significant. All statistical analyses were performed using the IBM Statistical Package for the Social Sciences (SPSS) version 24 (IBM Corp., Armonk, NY).

\section{Results}

A total of 50 COVID-19 positive patients were included in the study. The mean age was $41.70+7.106$. The serum ferritin levels were measured by the chemiluminescence assay (CLIA). Seventy-four percent (74\%) of the participants are males, and $34 \%$ are females. Forty-six percent $(46 \%)$ of the participants are between the ages of 45 and 50 . Twenty-eight percent (28\%) of the patients have co-morbidities, in which a combination of hypertension and diabetes mellitus is the most common. The serum ferritin levels of 29 patients (58\%) are greater than 300. Forty-seven patients (94\%) recovered, and three patients (6\%) died during the study, which was regarded as the final outcome (Table 1). 


\section{Cureus}

\begin{tabular}{|c|c|c|c|}
\hline & & No. of. Individuals & Percentage $\%$ \\
\hline \multirow{2}{*}{ Gender } & Male & 37 & $74 \%$ \\
\hline & Female & 13 & $26 \%$ \\
\hline \multirow{3}{*}{ Age } & $25-35$ & 12 & $24 \%$ \\
\hline & $36-44$ & 15 & $30 \%$ \\
\hline & $45-50$ & 23 & $46 \%$ \\
\hline \multirow{5}{*}{ Co-morbidities } & Only hypertension & 3 & $6 \%$ \\
\hline & Only diabetes mellitus & 5 & $10 \%$ \\
\hline & Hypertension and diabetes & 4 & $8 \%$ \\
\hline & Hyperthyroid & 1 & $2 \%$ \\
\hline & Hypothyroid & 1 & $2 \%$ \\
\hline \multirow{2}{*}{ Serum ferritin levels } & Less than/equal to 300 & 21 & $42 \%$ \\
\hline & Greater than 300 & 29 & $58 \%$ \\
\hline \multirow{2}{*}{ Final outcome } & Died & 3 & $6 \%$ \\
\hline & Recovered & 47 & $94 \%$ \\
\hline
\end{tabular}

TABLE 1: Demographic details, serum ferritin levels, and the final outcome of the patients $(n=50)$

The recovery rate (94\%) was very high and is a good sign of COVID-19. Low levels of serum ferritin were observed among them, where treatment with vitamin $\mathrm{C}$ and dexamethasone as key modalities. The mean serum ferritin levels among patients who survived and those who expired were $478.81 \mathrm{ng} / \mathrm{ml}$ and $1410 \mathrm{ng} / \mathrm{ml}$, respectively (Table 2).

\begin{tabular}{|l|l|l|}
\hline & Recovered $(\mathrm{n}=\mathbf{4 7})$ (Mean + SD) & Death ( $\mathrm{n}=\mathbf{3})$ (Mean + SD) \\
\hline Serum ferritin levels $(\mathrm{ng} / \mathrm{ml})$ & $478.81+424.69$ & $1410+370.7$ \\
\hline
\end{tabular}

TABLE 2: Mean and $p$-values of serum ferritin among recovered and death patients $(n=50)$

\section{Discussion}

Ferritin (storage protein) is involved in iron metabolism, which contains $\mathrm{L}$ and $\mathrm{H}$ subunits expressed in the lung and heart, respectively. The $\mathrm{H}$ subunit involves the inflammatory mechanism by participating in myeloid and lymphoid cell proliferation and stimulating TIM-2, a specific ferritin receptor. H-ferritin plays a major role in immunomodulatory and pro-inflammatory activities by activating several inflammatory mediators such as IL- $1 \beta$. Ferritin was found only in the lymph node B area, indicating its role as an antigen, which stimulates macrophage activation related to hyperferritinemia [8].

Previous research studies have revealed the role of hyperferritinemia in assessing the severity of disease among COVID-19 patients. The possible mechanisms include increased ferritin synthesis by proinflammatory cytokines such as tumor necrosis factor-alpha (TNF- $\alpha$ ), interleukin 6 (IL-6), and IL-1 $\beta$. This leads to increased inflammation that causes cell damage and the release of ferritin [9]. Hyperferritinemia patients were found to have increased mortality risk; it is not clear whether it is a coincidence or due to viral pathogenesis [10].

Through this observational study, we focused on the activities of serum ferritin as a vital SARS-CoV-2 infection marker among COVID-19 patients. The serum activities of ferritin were markedly increased in the patients who could not survive the treatment as compared with patients who finally recovered from the infection. This could be attributed to more severe secondary bacterial infection and increased inflammation leading to exacerbated COVID-19. The reasons for increased ferritin can be due to viral or bacterial infection, hemochromatosis, and long-term transfusion [11]. When secondary microbial infections occur 
alongside a pre-existing viral infection, an increase in the serum activities of ferritin was commonly observed, which was attributed to the release of iron from the reticuloendothelial system [12].

Patients with bacterial infection are found to have higher ferritin levels as compared to patients with a viral infection, which is evident through some previous studies [13]. In addition, the elevation of serum ferritin levels is predictive of a poor outcome in hospitalized patients with influenza infection [14]. In the present study, almost all COVID-19 patients showed increased serum ferritin levels, but the activities of serum ferritin extremely increased in the severely ill COVID-19 group, who could not survive. The increased ferritin might indicate a severe secondary bacterial infection in COVID-19 and might be utilized as a marker of poor prognosis [15].

From a randomized controlled trial in critically ill patients with sepsis-induced acute respiratory distress syndrome (ARDS) ( $\mathrm{n}=167$ ), it is observed that the administration of intravenous (IV) vitamin C $200 \mathrm{mg} / \mathrm{kg}$ per day for four days lowered 28 -day mortality in the treatment group ( $29.8 \%$ vs. $46.3 \%$; $\mathrm{P}=0.03$ ), coinciding with more days alive and free of the hospital and intensive care unit (ICU) admission [16]. From a study conducted in patients hospitalized with COVID-19, the use of dexamethasone resulted in a 28-day decreased mortality among those who were receiving either invasive mechanical ventilation or oxygen alone at randomization [17]. The recovery rate in this study seems to be high due to treatment with a high dose of vitamin $\mathrm{C}$ and dexamethasone and because none of the patients recruited in the study were $>65$ years of age, a well-established predisposing factor for severe and life-threatening COVID-19.

\section{Limitations of the study}

The major concern this study had was the absence of a comparator or a control group. The outcome of the patients could not be attributed either to the activities of serum ferritin or the treatment regimen that included dexamethasone and vitamin C, which was given universally for all the COVID-19 patients.

\section{Conclusions}

Increased activities of serum ferritin were observed among most COVID-19 patients. The serum activities of ferritin were extremely elevated among the COVID-19 patients who could not survive the treatment as compared to the recovered patients. The serum concentrations of ferritin could be used as a prognostic marker in the management of COVID-19 patients.

\section{Additional Information}

\section{Disclosures}

Human subjects: Consent was obtained by all participants in this study. PIMS Institutional Review Board issued approval IEC/PIMS/01/2020. Informed consent was taken from all patients, and confidentiality of information was assured to them. This study was approved by the Institutional Ethical Committee of Prathima Institute of Medical Sciences, Telangana. Animal subjects: All authors have confirmed that this study did not involve animal subjects or tissue. Conflicts of interest: In compliance with the ICMJE uniform disclosure form, all authors declare the following: Payment/services info: All authors have declared that no financial support was received from any organization for the submitted work. Financial relationships: All authors have declared that they have no financial relationships at present or within the previous three years with any organizations that might have an interest in the submitted work. Other relationships: All authors have declared that there are no other relationships or activities that could appear to have influenced the submitted work.

\section{References}

1. Suvvari TK, Kutikuppala LV, Babu GK, Jadhav M: Understanding the unusual viral outbreak: coronavirus disease 2019. J Curr Res Sci Med. 2020, 6:3-10. 10.4103/jcrsm.jcrsm_30_20

2. Pal M, Berhanu G, Desalegn C, et al.: Severe acute respiratory syndrome coronavirus-2 (SARS-CoV-2): an update. Cureus. 2020, 12:e7423. 10.7759/cureus.7423

3. Abbaspour N, Hurrell R, Kelishadi R: Review on iron and its importance for human health. Research J Med Sci. 2014, 19:164-174.

4. Renfei Lu, Jianru Qin, Yan Wu, et al.: Epidemiological and clinical characteristics of COVID-19 patients in Nantong, China. J Infect Dev Ctries. 2020, 14:440-446. 10.3855/jidc.12678

5. Tao Liu, Jieying Zhang, Yuhui Yang, et al.: The potential role of IL-6 in monitoring severe case of coronavirus disease 2019 [PREPRINT]. medRxiv. 2020, 10.1101/2020.03.01.20029769

6. Son NE: Influence of ferritin levels and inflammatory markers on HbA1c in the type 2 diabetes mellitus patients. Inflammatory markers on HbA1c in the type 2 diabetics. Pak J Med Sci. 2019, 35:1030-1035. 10.12669/pjms.35.4.1003

7. Chen N, Zhou M, Dong X, et al.: Epidemiological and clinical characteristics of 99 cases of 2019 novel coronavirus pneumonia in Wuhan, China: a descriptive study. Lancet. 2020, 395:507-513. 10.1016/S01406736(20)30211-7

8. R.G. Ruddell, D. Hoang-Le, J.M. Barwood, et al.: Ferritin functions as a pro-inflammatory cytokine via ironindependent protein kinase $\mathrm{C}$ zeta/ nuclear factor kappaB-regulated signaling in rat hepatic stellate cells. Hepatology. 2009, 49:887-900. 


\section{Cureus}

9. Kell DB, Pretorius E: Serum ferritin is an important inflammatory disease marker, as it is mainly a leakage product from damaged cells. Metallomics. 2014, 6:748-773. 10.1039/c3mt00347g

10. Edeas M, Saleh J, Peyssonnaux C: Iron: Innocent bystander or vicious culprit in COVID-19 pathogenesis? . Int J Infect Dis. 2020, 97:303-305. 10.1016/j.ijid.2020.05.110

11. Lalueza A, Ayuso B: Elevation of serum ferritin levels for predicting a poor outcome in hospitalized patients with influenza infection. Clin Microbiol Infect. 2020, 26:9-15. 10.1016/j.cmi.2020.02.018

12. Slaats J, Ten Oever J, van de Veerdonk FL, Netea MG: IL-1 $\beta /$ IL-6/CRP and IL-18/ferritin: distinct inflammatory programs in infections. PLoS Pathog. 2016, 12:1005973. 10.1371/journal.ppat.1005973

13. Kernan KF, Carcillo JA: Hyperferritinemia and inflammation. Int Immunol. 2017, 29:401-409. 10.1093/intimm/dxx031

14. Senjo H, Higuchi T, Okada S, Takahashi O: Hyperferritinemia: causes and significance in a general hospital . Hematology. 2018, 23:817-822. 10.1080/10245332.2018.1488569

15. Sanaei Dashti A, Alizadeh S, Karimi A, Khalifeh M, Shoja SA: Diagnostic value of lactate, procalcitonin, ferritin, serum-C-reactive protein, and other biomarkers in bacterial and viral meningitis: a cross-sectional study. Medicine. 2017, 96:e7637. 10.1097/MD.0000000000007637

16. Fowler AA III, Truwit JD, Hite RD, et al.: Effect of vitamin C infusion on organ failure and biomarkers of inflammation and vascular injury in patients with sepsis and severe acute respiratory failure. The CITRISALI randomized clinical trial. JAMA. 2019, 322:1261-1270. 10.1001/jama.2019.11825

17. Horby P, Lim WS, Emberson J, et al.: Dexamethasone in hospitalized patients with COVID-19: preliminary report. N Engl J Med. 2020, [Epub ahead of print]: 10.1056/NEJMoa2021436 